Rev. Adm. Saúde Vol. 17, № 67, Abr. - Jun. 2017

http://dx.doi.org/10.23973/ras.67.25

RELATO DE CASO

\title{
Implantação do Programa CQH no Hospital Regional de Quiche e em outros cinco hospitais na República da Guatemala
}

Implementation of the CQH Program at the Regional Hospital de Quiche and in five other hospitals in the Republic of Guatemala

José Manoel de Camargo Teixeira ${ }^{1}$, Milton M. Osaki ${ }^{2}$

1. Médico, administrador hospitalar e de sistemas de saúde, ex-diretor do PROAHSA

2. Médico, administrador hospitalar e de sistemas de saúde, consultor da JICA

\section{RESUMO}

Neste artigo são relatadas as ações para a implantação do Programa CQH em hospitais da Guatemala.

O PROAHSA (Programa de Estudos Avançados em Administração Hospitalar e Sistemas de Saúde) do Hospital das Clínicas da Faculdade de Medicina da Universidade de São Paulo, patrocinado pela JICA (Japan International Cooperation Agency), agência de cooperação do governo japonês, desenvolveu ações para implantar o Programa CQH no Hospital Regional de Quiché (HRQ) no Departamento de Quiche e em mais cinco hospitais da Guatemala.

As ações deste projeto basearam-se na realização de avaliações dentro do modelo $\mathrm{CQH}$ e na capacitação de 15 gestores do $\mathrm{HRQ}$ em fundamentos e critérios que embasam o Programa CQH e em conceitos de administração hospitalar e gestão de sistemas de saúde.

O projeto foi denominado "Fortalecimento de Capacidades para o Manejo de Sistemas em Gerenciamento Hospitalar" e foi executado no período de julho de 2014 a julho de 2016. 
Palavras-chave: Administração hospitalar e sistemas de saúde. Modelo de gestão.

\begin{abstract}
In this paper, the actions for the implementation of the CQH Program in hospitals in Guatemala are reported.
\end{abstract}

The PROAHSA (Program of Advanced Studies in Hospital Administration and Health Systems) of the Hospital das Clínicas of the Faculty of Medicine of the University of São Paulo, sponsored by JICA (Japan International Cooperation Agency), developed a program to implement the $\mathrm{CQH}$ Program at the Quiché Regional Hospital (HRQ) in the Quiche Department and in five other hospitals in Guatemala.

The actions of this project were based on evaluations within the CQH model and training of $15 \mathrm{HRQ}$ managers on fundamentals and criteria that underpin the CQH Program and concepts of hospital administration and health systems management.

The project was called "Capacity Building for Management of Systems in Hospital Management" and was executed from July 2014 to July 2016.

Keywords: Hospital administration and health systems. Management model

\title{
INTRODUÇÃO
}

A República da Guatemala conta com uma extensão de $108.889 \mathrm{~km}^{2}$ dividida em 22 departamentos. Em 2006, contava com uma população de 14.361 .666 habitantes: $51,23 \%$ mulheres e $48,76 \%$ homens, segundo o Instituto Nacional de Estatística da Guatemala. Naquele ano, a densidade populacional era de 117 habitantes por $\mathrm{km}^{2}$; a população indígena correspondia a $41 \%$ da população; $51 \%$ da população total encontrava-se abaixo da linha de pobreza e $15,2 \%$ encontrava-se em situação de pobreza extrema. Cerca de $51,5 \%$ vivia em áreas rurais e $23 \%$ vivia na região metropolitana. De acordo com a Organização Panamericana de Saúde (OPAS), em 2009 a esperança de vida era de 70,5 anos, sendo 67,1 anos para os homens e 74,2 anos para as mulheres.

O sistema de saúde da Guatemala ${ }^{4}$ é composto por um setor público e outro privado. O setor público compreende o Ministério de Saúde Pública e Assistência Social (MSPAS), responsável por assistir $70 \%$ da população. $O$ Instituto Guatemalteco do Seguro Social (IGSS) oferece cobertura a $17,45 \%$ da 
população vinculada a emprego formal e a Sanidad Militar cobre os membros das forças armadas e a polícia, incluindo as suas famílias.

O setor privado atende cerca de $12 \%$ dos guatemaltecos na assistência à saúde. Neste setor destacam-se as organizações da sociedade civil com fins lucrativos e organizações religiosas sem fins lucrativos. O setor de seguros de saúde tem pouca participação.

Os recursos do Ministério da Saúde são resultantes de arrecadação federal, empréstimos, doações internacionais e taxas de serviço.

Embora a constituição da Guatemala estabeleça o direito à saúde e segurança social a todos os cidadãos, a cobertura real alcança apenas $48 \%$ da população.

Os gastos com saúde representam 7,1\% do PIB da Guatemala. O gasto per capita gira em torno de US\$337.

O MSPAS conta com uma rede de 43 hospitais de referência secundária e terciária, sendo dois de referência nacional, 7 especializados, 7 nacionais regionais, 14 departamentais e 13 distritais que atendem a cerca de 6.600 .000 pessoas. O MSPAS mantém ainda 3301 centros comunitários, 957 postos de saúde e 20 maternidades.

Estatísticas de 2009 mostram que o setor privado contava com 2287 clinicas médicas particulares e 62 hospitais.

A Organização Mundial da Saúde mostrou em 2005 que a Guatemala contava com 7 leitos por 10.000 habitantes enquanto El Salvador contava com 9; a Bolívia, com 10; o Peru, com 11 e a Colômbia, com 12 leitos. Ainda segundo a OMS, a Guatemala contava com 0,9 médicos por 1000 habitantes enquanto a Bolívia e Paraguai contavam com 1,2. No Departamento de Quiché o índice era de 0,1 .

Em 2009, o Colégio de Médicos e Cirurgiões da Guatemala registrava 12.940 médicos no país. Na Guatemala existem cinco escolas médicas, sendo apenas uma pública (Universidade de São Carlos).

Segundo o MSPAS, os principais problemas de saúde são a grande mortalidade materna, a mortalidade infantil, a desnutrição e as doenças infectocontagiosas.

O Departamento de Quiché, onde se desenvolveu o projeto, encontra-se situado na região nordeste da Guatemala. Pertence à conhecida Franja Transversal do Norte.

Este departamento, durante a guerra civil que viveu o país entre 1960 e 1996, foi cenário de combates violentos principalmente na área petroleira de Ixcán.

O município de Santa Cruz del Quiché, capital do departamento, não contava com serviço de saúde até 1944, quando ocorreu a adequação de uma construção para atendimento de pacientes, dando-se o nome de Hospital Nacional Santa Helena. 
Em 1954, em terreno contíguo, foram construídas novas instalações da unidade de saúde e também uma capela dedicada à Santa Helena.

No ano de 1958, houve a compra do terreno de aproximadamente 2600 metros quadrados, vizinho à capela Santa Helena, para ampliação do hospital, que foi concluído em 1962.

Na década de 70, grande parte dos atendimentos eram demandas cirúrgicas por ferimentos de armamentos de guerra e o hospital chegou a ter atendimento compatível com $200 \%$ de ocupação. Em 1972, foram construídas salas cirúrgicas para os referidos atendimentos.

O hospital atendia neste período quatro especialidades: clínica médica, clínica cirúrgica, pediatria e ginecologia/obstetrícia.

Em março de 1982, um grupo de médicos, liderado pelo Dr. Guillermo Crocker Córdova, levou ao presidente da república a necessidade de um novo hospital. O pedido foi encaminhado ao Banco Interamericano de Desenvolvimento. A aprovação da demanda ocorreu em agosto de 1983.

Entre 1985 e 1986, foram realizados estudos para a construção da instituição, cuja pedra fundamental foi lançada em julho de 1987. Em 1988, foi iniciada a construção e em maio de 1995, foram transferidos para a nova estrutura os 150 leitos que funcionavam no antigo Hospital Santa Helena.

A inauguração do novo hospital ocorreu em 5 de junho de 1995, com 222 leitos.

Em setembro de 2013, o Ministério da Saúde elevou a classificação do hospital e este se tornou referência terciária do Departamento de Quiché, sendo renomeado como Hospital Regional de Quiché.

Atualmente o hospital tem 171 leitos distribuídos da maneira mostrada na Tabela 1.

Tabela 1. Distribuição e quantidades de leitos do HRQ.

\begin{tabular}{|l|r|}
\hline Especialidade & Leitos \\
\hline Clínica médica - homens & 11 \\
\hline Clínica médica - mulheres & 11 \\
\hline Clínica cirúrgica - homens & 14 \\
\hline Clínica cirúrgica - mulheres & 14 \\
\hline Ortopedia/traumatologia - homens & 12 \\
\hline Ortopedia/traumatologia - mulheres & 7 \\
\hline Obstetrícia & 37 \\
\hline Ginecologia & 4 \\
\hline Pediatria & 13 \\
\hline Recuperação nutricional & 5 \\
\hline Neonatologia (patológico) & 10 \\
\hline Parto canguru & 2 \\
\hline
\end{tabular}




\begin{tabular}{|l|r|}
\hline Resguardo obstétrico & 3 \\
\hline Clínica cirúrgica - pediatria & 4 \\
\hline Ortopedia/traumatologia - pediatria & 4 \\
\hline Emergência pediátrica (observação) & 5 \\
\hline UTI pediátrica & 7 \\
\hline Emergência adultos (observação) & 3 \\
\hline UTI adultos & 5 \\
\hline \multicolumn{2}{|c|}{ Total de leitos: } \\
\hline
\end{tabular}

O Hospital Regional de Quiché conta ainda com serviços de odontologia, cirurgia ambulatorial, fisioterapia e reabilitação, nutrição, banco de sangue, banco de leite, laboratório de análises clínicas, diagnóstico por imagens (radiologia, mamografia, ultrassonografia e ecocardiografia), farmácia e nutrição.

Em 07 de outubro de 2014, o Hospital Regional de Quiché recebeu a certificação de Hospital Amigo do Aleitamento Materno do Fundo das Nações Unidas para a Infância.

\section{RELATO DO DESENVOLVIMENTO DO PROJETO}

O projeto decorreu da percepção de que o Hospital Regional de Quiché necessitava fortalecer seu sistema de gestão para implantar um sistema de informática.

Em setembro de 2012, o HRQ recebeu em doação o sistema de informações S4 utilizado nos hospitais da Secretaria de Estado da Saúde de São Paulo.

Foi iniciado então o projeto "Apoio Técnico para a Gestão Hospitalar Baseado em um Sistema de Informações" para implementar este sistema de informações.

No decorrer dos trabalhos para a implantação do Sistema S4 percebeu-se que o Hospital Regional de Quiché necessitava fortalecer seu sistema de gestão para obter sucesso na implantação do sistema de informática.

Denominado projeto "Fortalecimento de Capacidades para o Manejo de Sistemas em Gerenciamento Hospitalar", os trabalhos iniciaram-se em Julho de 2014 e duraram dois anos.

O projeto tinha como principal objetivo a implantação do Programa CQH ${ }^{1,5}$ no Hospital Regional de Quiché e a disseminação para mais cinco hospitais. A estratégia seria: a) realizar avaliações e levantamentos com o objetivo de melhorar as fragilidades administrativas e b) realizar a capacitação de 15 gestores do HRQ por meio da prática dos fundamentos e critérios do programa.

Os 15 gestores do HRQ selecionados para os treinamentos eram divididos em 5 médicos, 5 enfermeiras e 5 gestores administrativos. Na Tabela 2 constam os nomes e os setores dos participantes. 


\section{Tabela 2. Nomes e setores de trabalho dos 15 gestores do HRQ selecionados para a capacitação.}

\begin{tabular}{|l|l|}
\hline Nome do gestor & Setor de trabalho \\
\hline Dra. Ana Virginia Acifuina Arriaga & Clínica médica \\
\hline Dra. Ligia Raquel Terre Prado & Pediatria \\
\hline Dr. Joaquim Palma Canizales & Anestesiologia \\
\hline $\begin{array}{l}\text { Dra. Karen del Rosario Barrientos } \\
\text { Trujillo }\end{array}$ & Ginecologia/obstetrícia \\
\hline Dr. Hector Anotnio Gonzalez Cajas & Epidemiologia \\
\hline EP Cláudia Yaneth Pineda Rodas & Enfermagem - centro cirúrgico \\
\hline EP Basilia Noemi Bala Lopez & Enfermagem - coordenação \\
\hline EP Sandra Patricia Lopez Soto & Enfermagem - emergência \\
\hline EP Edilia Yesenia Curruchiche Lopez & Enfermagem - UTI \\
\hline Cristian Leonel Gonzales Sapon & SAME \\
\hline Valeska Gabriela Mendez Estrada & Contabilidade \\
\hline Eva Maribel Sapon Herrera & Gestão de pessoas \\
\hline Ileana Maribel Noriega Lainez & Farmácia \\
\hline Javier Francisco Barrios Cifuentes & Radiologia \\
\hline Maria Angelica Tiquiran Ramirez & Enfermagem - Coordenadora do \\
& Projeto no HRQ \\
\hline
\end{tabular}

O modelo CQH de gestão hospitalar é um conjunto de fundamentos e critérios que permitem avaliar a qualidade do atendimento médico-hospitalar, baseado no registro, na análise de dados, na aferição da adequação dos serviços em conformidades com as suas normas e critérios.

O Programa CQH foi inspirado nos trabalhos da Comissão Conjunta de Acreditação de Organização de Saúde (CCOS) dos EUA nos anos 1989 e 1990. Desde 1991, está estabelecida na sede da Associação Paulista de Medicina e trata-se de uma atividade de responsabilidade social desta entidade. O CQH é um programa de adesão voluntária, cujo objetivo é contribuir para a melhoria contínua da qualidade hospitalar. Estimula a autoavaliação das instituições hospitalares estabelecendo um componente educacional muito importante, que é o incentivo à mudança de atitude e de comportamento. Preconiza o trabalho coletivo, envolvendo grupos multidisciplinares no aprimoramento dos processos de atendimento. A missão do programa é contribuir para a melhoria contínua da qualidade do atendimento nos serviços de saúde mediante metodologia específica, estimulando a melhoria contínua da qualidade de produtos e serviços, estabelecendo amplo entendimento dos requisitos de excelência e desempenho.

As capacitações foram desenvolvidas por especialistas em administração hospitalar e de sistemas de saúde coordenados pelo PROAHSA ${ }^{2}$.

O PROAHSA é formado a partir de um convênio entre o Hospital das Clínicas da Faculdade de Medicina da Universidade de São Paulo, a Escola de Administração de Empresas de São Paulo da Fundação Getúlio Vargas e a Faculdade de Medicina da Universidade de São Paulo. Os principais objetivos 
deste programa são: desenvolvimento de programas de ensino, pesquisa, preparação de publicações e assistência técnica, no campo da administração hospitalar e de sistemas de saúde.

Os trabalhos desenvolvidos foram patrocinados pela Japan International Cooperation Agency (JICA) ${ }^{3}$.

A JICA é o órgão do Governo Japonês responsável pela implementação da Assistência Oficial para o Desenvolvimento (ODA), programa que apóia o crescimento e a estabilidade socioeconômica dos países em desenvolvimento, com o objetivo de contribuir para a paz e o desenvolvimento da sociedade internacional. Com uma rede de escritórios que se estende por quase 100 países, a JICA presta assistência a mais de150 países no mundo todo.

\section{Avaliações no Hospital Regional de Quiché}

Foram realizadas cinco avaliações no $H R Q$ com base no roteiro de avaliação de 100 pontos do Programa CQH. Uma das avaliações (a primeira) decorreu na forma de autoavaliação.

O roteiro de avaliação de 100 pontos tem como base os critérios que compõem o Modelo CQH de gestão hospitalar. O resultado da avaliação decorre do apontamento entre duas opções de resposta: $\operatorname{sim}(\mathrm{s})$ e não (n). No caso do hospital não oferecer o serviço, a condição será "não se aplica" (na). Os campos dos subitens deverão ser preenchidos com sim (s), não (n) ou não se aplica (na).

Assim, exemplificando, no critério Liderança a avaliação realiza-se através dos questionamentos:

1. O hospital tem organograma formalmente descrito e representativo da realidade organizacional? (s) (n)

2. O hospital tem valores e conceitos organizacionais formalmente definidos (missão, visão e políticas organizacionais)? (s) (n)

3. Os valores e conceitos organizacionais são de conhecimento das pessoas que compõem a força de trabalho e outras partes interessadas, quando pertinente? (s) (n)

A positividade ao questionamento traduz-se em pontuação.

A Tabela 3 demonstra a pontuação máxima possível por critério.

Tabela 3. Critérios do Roteiro de Avalição do Programa CQH e respectivas pontuações. 


\begin{tabular}{|c|c|}
\hline Critério & $\begin{array}{r}\text { Pontuação } \\
\text { Máxima }\end{array}$ \\
\hline P. Perfil & 5 \\
\hline I. Liderança & 10 \\
\hline 1.1 Governabilidade Corporativa & 3 \\
\hline 1.2 Exercício de Liderança e Promoção da Cultura de Excelência & 4 \\
\hline 1.3 Análise de Desempenho do Hospital & 3 \\
\hline II. Estratégias e Planos & 4 \\
\hline 2.1 Formulação de Estratégias & 2 \\
\hline 2.2 Implementação de Estratégias & 2 \\
\hline III. Clientes & 5 \\
\hline 3.1 Imagem e Conhecimento do Mercado & 3 \\
\hline 3.2 Relação com os Clientes & 2 \\
\hline IV. Sociedade & 5 \\
\hline 4.1 Responsabilidade Sócio Ambiental & 3 \\
\hline 4.2 Desenvolvimento Social & 2 \\
\hline V. Informações e Conhecimentos & 10 \\
\hline 5.1 Informação do Hospital & 5 \\
\hline 5.2 Informação Comparativa & 4 \\
\hline 5.3 Ativos Intangíveis e Conhecimento Organizacional & 1 \\
\hline VI. Pessoas & 10 \\
\hline 6.1 Sistema de Trabalho & 3 \\
\hline 6.2 Capacitação e Desenvolvimento & 4 \\
\hline 6.3 Qualidade de Vida & 3 \\
\hline VII. Processos & 11 \\
\hline 7.1 Processos Principais do Negócio e Processos de Apoio & 6 \\
\hline 7.2 Processo de Relação com os Fornecedores & 2 \\
\hline 7.3 Processo Econômico Financeiro & 3 \\
\hline VIII. Resultados & 40 \\
\hline 8.1 Resultados Econômico Financeiros & 8 \\
\hline 8.2 Resultados Relativos a Clientes e Mercado & 10 \\
\hline 8.3 Resultados Relativos à Sociedade & 2 \\
\hline 8.4 Resultados Relativos a Pessoas & 8 \\
\hline $\begin{array}{l}\text { 8.5 Resultados dos Processos Principais do Negocio e dos Processos de } \\
\text { Apoio }\end{array}$ & 10 \\
\hline 8.6 Resultados Relativos com os Fornecedores & 2 \\
\hline Total & 100 \\
\hline
\end{tabular}

\section{Resultados das Avaliações}

Como inicio das atividades do projeto orientou-se a realização de autoavaliação pelos 15 gestores do HRQ. Esta atividade foi desenvolvida em outubro de 2013 e o resultado foi de 77,04 pontos. 
Em julho de 2014, ocorreu a primeira avaliação do $H R Q$ realizada pelas avaliadoras do Programa CQH, Neuza K.Uchiyama e Cibelle Naves. Nesta avaliação, o Hospital Regional de Quiché obteve 49,18 pontos.

As mesmas avaliadoras que realizaram a primeira avaliação desenvolveram em julho de 2015 a segunda avaliação. Nesta avaliação o HRQ obteve o resultado de 64,57 pontos.

Em fevereiro de 2016, foi realizada a terceira avaliação ao $H R Q$ pelas avaliadoras Cibelle Naves e Isabel Dias, com resultado de 59,21 pontos.

Em junho de 2016 as avaliadoras Neuza K. Uchiyama e Cibelle Naves realizaram a quarta avaliação e o Hospital Regional de Quiché obteve o resultado de 60,80 pontos.

A Tabela 4 apresenta todos os resultados das avaliações realizadas no HRQ.

Tabela 4. Pontuações das avaliações feitas no HRQ.

\begin{tabular}{|c|c|c|c|c|c|c|c|}
\hline Critério & & $\begin{array}{l}\text { Pontuação } \\
\text { Máxima }\end{array}$ & $\begin{array}{l}\text { Auto } \\
\text { avaliação } \\
\text { Outubro } \\
2013\end{array}$ & $\begin{array}{l}\text { Primeira } \\
\text { Avaliação } \\
\text { Julho } 2014\end{array}$ & $\begin{array}{l}\text { Segunda } \\
\text { Avaliação } \\
\text { Julho } 2015\end{array}$ & $\begin{array}{l}\text { Terceira } \\
\text { Avaliação } \\
\text { Fevereiro } \\
2016\end{array}$ & $\begin{array}{l}\text { Quarta } \\
\text { Avaliação } \\
\text { Junho } 2016\end{array}$ \\
\hline Perfil & $P$ & 5 & 3,86 & 2,27 & 2,84 & 2.73 & 3.07 \\
\hline \multirow[t]{3}{*}{ Liderança } & 1.1 & \multirow[t]{3}{*}{10} & 2,31 & 2,00 & 2,54 & 2,31 & 2.54 \\
\hline & 1.2 & & 4,00 & 2,67 & 2,80 & 2,40 & 2.40 \\
\hline & 1.3 & & 3,00 & 1,88 & 2,67 & 2,67 & 2,67 \\
\hline \multirow{2}{*}{$\begin{array}{l}\text { Estratégias e } \\
\text { Planos }\end{array}$} & 2.1 & \multirow[t]{2}{*}{4} & 1,50 & 1,71 & 2,00 & 1,75 & 2,00 \\
\hline & 2.2 & & 1,60 & 0,22 & 0,00 & 0,20 & 0,00 \\
\hline \multirow[t]{2}{*}{ Clientes } & 3.1 & \multirow[t]{2}{*}{5} & 3,00 & 2,00 & 2,40 & 2,40 & 2,70 \\
\hline & 3.2 & & 1,78 & 1,50 & 1,78 & 1,78 & 1,78 \\
\hline \multirow[t]{2}{*}{ Sociedade } & 4.1 & \multirow[t]{2}{*}{5} & 2,67 & 2,25 & 2,33 & 2,33 & 2,33 \\
\hline & 4.2 & & 2,00 & 2,00 & 2,00 & 2,00 & 2,00 \\
\hline \multirow{3}{*}{$\begin{array}{l}\text { Informações e } \\
\text { Conhecimentos }\end{array}$} & 5.1 & \multirow[t]{3}{*}{10} & 5,00 & 4,29 & 4,09 & 4,09 & 4,32 \\
\hline & 5.2 & & 4,00 & 1,33 & 4,00 & 4,00 & 4,00 \\
\hline & 5.3 & & 0,67 & 0,38 & 0,44 & 0,56 & 0,89 \\
\hline \multirow[t]{3}{*}{ Pessoas } & 6.1 & \multirow[t]{3}{*}{10} & 2,70 & 2,33 & 2,40 & 1,80 & 2,10 \\
\hline & 6.2 & & 4,00 & 2,80 & 3,27 & 2,55 & 2,91 \\
\hline & 6.3 & & 1,80 & 0,32 & 1,50 & 1,35 & 0,90 \\
\hline
\end{tabular}




\begin{tabular}{|c|c|c|c|c|c|c|c|}
\hline \multirow[t]{3}{*}{ Processos } & 7.1 & \multirow[t]{3}{*}{11} & 5,26 & 3,80 & 4,09 & 4,38 & 4,25 \\
\hline & 7.2 & & 1,43 & 0,33 & 1,43 & 1,33 & 1,67 \\
\hline & 7.3 & & 2,54 & 2,25 & 2,08 & 2,31 & 1,85 \\
\hline \multirow[t]{6}{*}{ Resultados } & 8.1 & \multirow[t]{6}{*}{40} & 2,67 & 5,33 & 5,33 & 2,67 & 2,67 \\
\hline & 8.2 & & 8,33 & 1,67 & 5,00 & 3,33 & 5,00 \\
\hline & 8.3 & & 1,43 & 1,14 & 1,43 & 1,14 & 1,71 \\
\hline & 8.4 & & 4,00 & 1,00 & 1,00 & 2,00 & 1,00 \\
\hline & 8.5 & & 7,50 & 3,75 & 7,14 & 7,14 & 5,71 \\
\hline & 8.6 & & 0,00 & 0,00 & 0,00 & 0,00 & 0,33 \\
\hline Total & & 100 & 77,04 & 49,22 & 64,57 & 59,21 & 60,80 \\
\hline
\end{tabular}

O resultado de 77,04 pontos da autoavaliação não traduzia a qualidade do atendimento médico-hospitalar observada no HRQ e estava associado à incompreensão da metodologia.

O resultado da primeira avaliação do Hospital Regional de Quiché de 49,22 pontos demonstrou o real nível de qualidade observado na instituição. Eram evidentes as oportunidades de melhoria nos processos de gestão, atendimento médico-hospitalar e estrutura física.

Da primeira avaliação para a avaliação seguinte observou-se expressivo aumento de $31 \%$ dos pontos. Credita-se o incremento da pontuação ao treinamento realizado em setembro de 2014. Foram perceptíveis as melhoras nos processos de gestão e nas instalações físicas com consequente melhora na assistência. Ocorreram melhoras visíveis na limpeza com a ocorrência de reformas físicas (UTI e enfermarias). Houve também construção de espaço físico para alojar familiares dos pacientes.

O resultado da terceira avaliação mostrou decréscimo de 8,31\% na pontuação em relação à avaliação anterior. Esta queda no resultado é explicada pela nota zero observada no critério Resultados relacionado ao subcritério Fornecedores. Este retrocesso pode ser associado à irregularidade e inconstância nos trabalhos de consolidação do modelo neste período. $O$ fato da instituição não apresentar conformidade em um item que na avaliação anterior havia apresentado conformidade demonstrou instabilidade nos trabalhos de consolidação do modelo. Esta queda na pontuação pode ainda ser explicada pela dificuldade observada neste período de interação entre alguns gestores participantes do projeto. Verificou-se falha no entrosamento entre alguns gestores que afetou o desempenho das tarefas do projeto. Este desencontro culminou com a vinda de apenas 10 gestores para a terceira capacitação.

Na quarta e última avaliação ocorrida em Junho de 2016 a marca atingida foi de 60,80 pontos, verificando-se aumento de $2,68 \%$ em relação à terceira avaliação. Este resultado pode ser creditado ao retorno de constância no 
desenvolvimento dos trabalhos de consolidação do modelo e também a melhor compreensão e aplicação efetiva dos conceitos do Modelo CQH. Outra explicação para a melhora do resultado está relacionada ao trabalho realizado em fevereiro de $2016 \mathrm{com}$ a ida de dois especialistas do PROAHSA, Antonio Carlos da Silva e Isabel Dias, que realizaram trabalho de diagnóstico situacional do $\mathrm{HRQ}$. Sobre as oportunidades de melhoria verificadas, desenvolveram-se ações na metodologia QUICK WIN.

\section{Capacitação dos 15 gestores do HRQ}

A primeira capacitação ocorreu no período de 1 a 13 de setembro de 2014 em São Paulo.

Neste primeiro treinamento o objetivo principal foi apresentar o modelo de gestão do programa $\mathrm{CQH}$. Enfatizaram-se os fundamentos e critérios do modelo de gestão considerando-se os resultados observados na autoavaliação realizada em outubro de 2013 e na avaliação feita em julho de 2014.

Nesta primeira capacitação foram também realizadas visitas aos hospitais do Programa CQH detentores do selo de conformidade para compreensão da prática dos critérios.

Como percalço durante o primeiro treinamento, pode ser registrada a comunicação do afastamento do Dr. Guilhermo Crocker Cordóva da direção geral do Hospital Regional de Quiché por decisão governamental. Este dirigente foi o incentivador da implantação do projeto. Este episódio marcou os ânimos dos participantes.

Durante o transcorrer do primeiro treinamento os gestores do HRQ foram recebidos na Associação Paulista de Medicina pelo presidente da instituição, Dr. Florisval Meinão.

Na Tabela 5 segue a programação e cronograma do treinamento.

Tabela 5. Cronograma da primeira capacitação realizada de 1 a 13 de setembro de 2014.

\begin{tabular}{|l|l|l|l|l|}
\hline Data & Horário & Atividade & Local & Palestrante \\
\hline $\mathbf{0 1 / 0 9}$ & $09: 00-12: 00$ & $\begin{array}{l}\text { Apresentação do } \\
\text { programa de visita e do } \\
\text { Projeto }\end{array}$ & Hotel Nikkey-SP & Haino Burmester \\
\hline & $\begin{array}{l}\text { Apresentação do } \\
\text { Modelo de Gestão }\end{array}$ & Hotel Nikkey -SP & $\begin{array}{l}\text { Ivana Mara } \\
\text { Rodrigues da Silva }\end{array}$ \\
\hline
\end{tabular}




\begin{tabular}{|c|c|c|c|c|}
\hline $02 / 09$ & $09: 00-12: 00$ & Liderança & Hotel Nikkey-SP & Haino Burmester \\
\hline & $14: 00-17: 00$ & $\begin{array}{l}\text { Planejamento } \\
\text { Estratégico }\end{array}$ & Hotel Nikkey-SP & $\begin{array}{l}\text { Fabio Pacheco } \\
\text { Muniz de Souza e } \\
\text { Castro }\end{array}$ \\
\hline \multirow[t]{2}{*}{$03 / 09$} & 09:00 - 12:00 & Clientes e Sociedade & Hotel Nikkey-SP & $\begin{array}{l}\text { José Agenor Mei } \\
\text { Silveira }\end{array}$ \\
\hline & $14: 00-17: 00$ & $\begin{array}{l}\text { Informação e } \\
\text { Conhecimento }\end{array}$ & Hotel Nikkey-SP & Haino Burmester \\
\hline \multirow[t]{2}{*}{$04 / 09$} & 09:00 - 12:00 & $\begin{array}{l}\text { Informação e } \\
\text { Conhecimento }\end{array}$ & Hotel Nikkey-SP & $\begin{array}{l}\text { Marco Antonio } \\
\text { Gutierrez }\end{array}$ \\
\hline & $14: 00-17: 00$ & Gestão de Pessoas & Hotel Nikkey-SP & $\begin{array}{l}\text { Maria Aparecida } \\
\text { Novaes }\end{array}$ \\
\hline \multirow[t]{2}{*}{ 05/09 } & $09: 00-12: 00$ & Gestão de Processos & Hotel Nikkey-SP & $\begin{array}{l}\text { Marcelo Marinho } \\
\text { Aidar }\end{array}$ \\
\hline & $14: 00-17: 00$ & Visita & $\begin{array}{l}\text { Hospital Santa Cruz- } \\
\text { SP }\end{array}$ & \\
\hline $06 / 09$ & & Programação livre & & \\
\hline $07 / 09$ & & Programação livre & & \\
\hline $\begin{array}{l}08 \text { e } \\
09 / 09\end{array}$ & $08: 30-17: 30$ & $\begin{array}{l}\text { Curso Implantando o } \\
\text { Modelo de Excelência } \\
\text { de Gestão no Setor } \\
\text { Saúde }\end{array}$ & $\begin{array}{l}\text { Hospital Policlin } 9 \text { de } \\
\text { Julho - SÃO JOSÉ } \\
\text { DOS CAMPOS }\end{array}$ & $\begin{array}{l}\text { Neusa Kyoko S. } \\
\text { Uchiyama e Cibelle } \\
\text { Naves de Oliveira }\end{array}$ \\
\hline $10 / 09$ & 08:30 - 17:30 & Visita & $\begin{array}{l}\text { Hospital Policlin } 9 \text { de } \\
\text { Julho - SÃO JOSÉ } \\
\text { DOS CAMPOS }\end{array}$ & $\begin{array}{l}\text { Neusa Kyoko S. } \\
\text { Uchiyama e Cibelle } \\
\text { Naves de Oliveira }\end{array}$ \\
\hline \multirow[t]{2}{*}{$11 / 09$} & 09:00 - 12:00 & Visita & $\begin{array}{l}\text { Instituto do Coração } \\
\text { do HCFMUSP-SÃO } \\
\text { PAULO }\end{array}$ & $\begin{array}{l}\text { Marco Antonio } \\
\text { Gutierrez }\end{array}$ \\
\hline & $14: 00-17: 00$ & Visita & $\begin{array}{l}\text { Instituto do Coração } \\
\text { do HCFMUSP-SÃOO } \\
\text { PAULO }\end{array}$ & $\begin{array}{l}\text { Marco Antonio } \\
\text { Gutierrez }\end{array}$ \\
\hline \multirow[t]{2}{*}{$12 / 09$} & 09:00 - 12:00 & Visita & $\begin{array}{l}\text { Fundação Nacional } \\
\text { da Qualidade-SÃO } \\
\text { PAULO }\end{array}$ & Jairo Martins \\
\hline & $15: 00-19: 00$ & Visita & $\begin{array}{l}\text { Hospital Vera Cruz } \\
\text { de Campinas- } \\
\text { CAMPINAS }\end{array}$ & Claudia Matias \\
\hline $13 / 09$ & & Programação livre & & \\
\hline
\end{tabular}


A segunda capacitação ocorreu em São Paulo no período de 8 a 18 de setembro de 2015.

Neste treinamento, além do reforço sobre os conceitos do Programa CQH, focaram-se os conceitos teóricos do Prêmio Nacional de Gestão em Saúde (PNGS) ${ }^{5}$. Foram desenvolvidas aulas para o preparo do relatório de gestão do PNGS.

Os gestores do $\mathrm{HRQ}$ foram treinados também para o uso de ferramentas de gestão como indicadores de desempenho para gestão de processos, pessoas, segurança do paciente e infecção hospitalar.

Foram ainda realizadas visitas a Hospitais do Programa CQH portadores do selo de conformidade.

A Tabela 6 detalha o cronograma e programação do segundo treinamento.

Tabela 6. Cronograma da segunda capacitação realizada de 8 a 18 de setembro de 2015.

\begin{tabular}{|c|c|c|c|}
\hline Data & Horário & Atividade & Palestrante \\
\hline \multirow{9}{*}{$\begin{array}{c}8 \mathrm{de} \\
\text { setembro } \\
\text { terca-feira }\end{array}$} & $\begin{array}{l}8 \mathrm{~h} 00 \\
8 \mathrm{~h} 00 \text { às } 8 \mathrm{~h} 30 \\
8 \mathrm{~h} 30 \text { às } 9 \mathrm{~h} 00\end{array}$ & $\begin{array}{l}\text { Recepção } \\
\text { Palestra JICA-SP - Japan Internacional Cooperation } \\
\text { Agency. } \\
\text { Abertura } \\
\text { Apresentação da programação }\end{array}$ & $\begin{array}{l}\text { Representante JICA-SP } \\
\text { Sr. Vicente Murakami } \\
\text { Dr. Haino Burmester } \\
\text { Dr. Milton Massayuki Qsaki } \\
\text { Dr. Massabiro Miyamoto } \\
\text { Enf }{ }^{\mathrm{B}} \text {. Neusa Kyoko Uchiyama } \\
\text { Enf. Cibelle Naves de Oliveira }\end{array}$ \\
\hline & $9 \mathrm{~h} 00$ às $10 \mathrm{~h} 30$ & $\begin{array}{l}\text { Apresentação do HRQ (produção, organograma, } \\
\text { personograma, processos, etc) } \\
\text { Mapa de Saúde do Departamento de Quiché e da } \\
\text { Guatemala }\end{array}$ & Equipe Guatemala \\
\hline & $10 \mathrm{~h} 30$ às $10 \mathrm{~h} 45$ & Coffee Break & \\
\hline & $10 \mathrm{~h} 45$ às $12 \mathrm{~h} 00$ & $\begin{array}{l}\text { Apresentação das atividades realizadas pelos } 15 \\
\text { gestores do HRQ de setembro/2014 a julho/2015 }\end{array}$ & Equipe Guatemala \\
\hline & $12 \mathrm{~h} 00$ às $14 \mathrm{~h} 00$ & Almoço & \\
\hline & $14 \mathrm{~h} 00$ às $14 \mathrm{~h} 50$ & Apresentação de cinco pôsteres; & Equipe Guatemala \\
\hline & 14 h50 às $16 \mathrm{~h} 30$ & Feedback Visita Realizada julho/2015 & $\begin{array}{l}\text { Enf's. Neusa Kyoko Uchiyama } \\
\text { Enf'. Cibelle Naves de Oliveira }\end{array}$ \\
\hline & $16 \mathrm{~h} 30$ às $16 \mathrm{~h} 45$ & Coffee Break & \\
\hline & $16 \mathrm{~h} 45$ às $18 \mathrm{~h} 00$ & Oficina de reflexão & $\begin{array}{l}\text { Dr. Haino Burmester } \\
\text { Dr. Milton Massayuki Qsaki } \\
\text { Dr. Massahiro Miyamoto } \\
\text { Enf }{ }^{\mathrm{g}} \text {. Neusa Kyoke Uchiyama } \\
\text { Enf }{ }^{\mathrm{a}} \text {. Cibelle Naves de Oliveira }\end{array}$ \\
\hline \multirow{3}{*}{$\begin{array}{l}9 \text { de } \\
\text { setembro } \\
\text { quarta-feira }\end{array}$} & $8 \mathrm{~h} 30$ às $12 \mathrm{~h} 00$ & $\begin{array}{l}\text { Modelo CQH - Compromisso com a Qualidade } \\
\text { Hospitalar } \\
\text { Revisão }\end{array}$ & $\begin{array}{l}\text { Dr. Antonio Eduardo Fernandes } \\
\text { D'Aguiar }\end{array}$ \\
\hline & $12 \mathrm{~h} 00$ às $14 \mathrm{~h} 00$ & Almoço & \\
\hline & $14 \mathrm{~h} 00$ às $18 \mathrm{~h} 00$ & PNGS - Prêmio Nacional de Gestão em Saúde & Enf ${ }^{a}$. Cibelle Naves de Oliveira \\
\hline \multirow{3}{*}{$\begin{array}{l}10 \text { de } \\
\text { setembro } \\
\text { quinta-feira }\end{array}$} & $8 \mathrm{~h} 30$ às $12 \mathrm{~h} 00$ & PNGS - Prêmio Nacional de Gestão em Saúde & Enf ${ }^{a}$ Cibelle Naves de Oliveira \\
\hline & $12 \mathrm{~h} 00$ às $14 \mathrm{~h} 00$ & Almoço & \\
\hline & $14 \mathrm{~h} 00$ às $18 \mathrm{~h} 00$ & PNGS - Prêmio Nacional de Gestão em Saúde & Enf ${ }^{\mathrm{B}}$ Cibelle Naves de Oliveira \\
\hline
\end{tabular}




\begin{tabular}{|c|c|c|c|}
\hline \multirow{3}{*}{$\begin{array}{c}11 \text { de } \\
\text { setembro } \\
\text { sexta-feira }\end{array}$} & $8 \mathrm{~h} 30$ às $12 \mathrm{~h} 00$ & $\begin{array}{l}\text { Curso de Redação do Relatório de Gestão - PNGS - } \\
\text { Prêmio Nacional de Gestão em Saúde }\end{array}$ & Prof. Marcelo Marinho Aidar \\
\hline & $12 \mathrm{~h} 00$ às $14 \mathrm{~h} 00$ & Almoço & \\
\hline & $14 \mathrm{~h} 00$ às $18 \mathrm{~h} 00$ & Técnicas de Comunicação & Dr. Rubens Baptista Junior \\
\hline \multirow{3}{*}{$\begin{array}{c}14 \text { de } \\
\text { setembro } \\
\text { segunda-feira }\end{array}$} & $8 \mathrm{~h} 30$ às $12 \mathrm{~h} 00$ & Indicadores de Desempenho & $\begin{array}{l}\text { Dra. Nancy Val y Val Peres da } \\
\text { Mota }\end{array}$ \\
\hline & $12 \mathrm{~h} 00$ às $14 \mathrm{~h} 00$ & Almoço & \\
\hline & $14 \mathrm{~h} 00$ às $18 \mathrm{~h} 00$ & Indicadores de Enfermagem & Enfa. Rosemeire Keiko Hangai \\
\hline \multirow{4}{*}{$\begin{array}{c}15 \text { de } \\
\text { setembro } \\
\text { terca-feira }\end{array}$} & $8 \mathrm{~h} 30$ às $12 \mathrm{~h} 00$ & Indicadores de Pessoas & Sra. Maria Aparecida Novaes \\
\hline & $12 \mathrm{~h} 00$ às $13 \mathrm{~h} 00$ & Almoço & \\
\hline & $13 \mathrm{~h} 00$ às $14 \mathrm{~h} 00$ & $\begin{array}{l}\text { Visita à EEP - Escola de Educação Permanente do } \\
\text { HCFMUSP }\end{array}$ & $\begin{array}{l}\text { Sra. Carolina Tavares } \\
\text { Canhisares. }\end{array}$ \\
\hline & $14 \mathrm{~h} 00$ às $18 \mathrm{~h} 00$ & Indicadores de Infecção Hospitalar & $\begin{array}{l}\text { Enf } f^{\mathrm{a}} \text { Cibelle Naves de Oliveira } \\
\text { Dra. Suzana Aparecida Silveira }\end{array}$ \\
\hline \multirow{3}{*}{$\begin{array}{c}16 \text { de } \\
\text { setembro } \\
\text { quarta-feira }\end{array}$} & $8 \mathrm{~h} 30$ às $12 \mathrm{~h} 00$ & Governança & Dr. Haino Burmester \\
\hline & $12 \mathrm{~h} 00$ às $14 \mathrm{~h} 00$ & Almoço & \\
\hline & $14 \mathrm{~h} 00$ às $18 \mathrm{~h} 00$ & Gestão de Risco e Segurança do Paciente & $\begin{array}{l}\text { Sra. Claudia Maria Figueiredo } \\
\text { Matias }\end{array}$ \\
\hline \multirow{3}{*}{$\begin{array}{c}17 \text { de } \\
\text { setembro } \\
\text { quinta-feira }\end{array}$} & $8 \mathrm{~h} 30$ às $12 \mathrm{~h} 00$ & Visita ao Instituto de Infectologia Emílio Ribas/SES/SP & $\begin{array}{l}\text { Dr. Milton Massayuki Qsaki } \\
\text { Enf. Jurini Valdisi da Silva } \\
\text { Dr. Anísio de Moura } \\
\text { Sra. Teresinha Passos Gotti }\end{array}$ \\
\hline & $12 \mathrm{~h} 00$ às $14 \mathrm{~h} 00$ & Almoço & \\
\hline & $14 \mathrm{~h} 00$ às $18 \mathrm{~h} 00$ & $\begin{array}{l}\text { Visita ao Instituto de Infectologia Emilio } \\
\text { Ribas/SES-SP }\end{array}$ & $\begin{array}{l}\text { Dr. Milton Massayuki Qsaki } \\
\text { Enf. Jurini Valdisi da Silva } \\
\text { Dr. Anísio de Moura } \\
\text { Sra. Teresinha Passos Gotti }\end{array}$ \\
\hline
\end{tabular}

\begin{tabular}{|c|c|c|c|}
\hline \multirow{5}{*}{$\begin{array}{c}18 \text { de } \\
\text { setembro } \\
\text { sexta-feira }\end{array}$} & $8 \mathrm{~h} 30$ às $12 \mathrm{~h} 00$ & $\begin{array}{l}\text { Evolução do roteiro de avaliação do Modelo CQH para } \\
\text { o PNGS }\end{array}$ & Sr. Antonio Carlos da Silva \\
\hline & $12 \mathrm{~h} 00$ às $14 \mathrm{~h} 00$ & Almoço & \\
\hline & $14 \mathrm{~h} 00$ às $16 \mathrm{~h} 00$ & Planejamento dos próximos passos & $\begin{array}{l}\text { Dr. José Manoel de Camargo } \\
\text { Teixeira } \\
\text { Dr. Haino Burmester } \\
\text { Dr. Milton Massayuki Qsaki }\end{array}$ \\
\hline & $16 \mathrm{~h} 00$ às $16 \mathrm{~h} 15 \mathrm{~h}$ & Coffee Break & \\
\hline & $16 \mathrm{~h} 15$ às $17 \mathrm{~h} 00$ & Entrega de certificados/Encerramento & $\begin{array}{l}\text { Dr. José Manoel de Camargo } \\
\text { Teixeira } \\
\text { Dr. Haino Burmester } \\
\text { Dr. Milton Massayuki Qsaki } \\
\text { Dr. Massabiro Miyamote } \\
\text { Enfa. Neusa Kyoko Uchiyama } \\
\text { Enfa Cibelle Naves de Oliveira }\end{array}$ \\
\hline
\end{tabular}

A terceira capacitação ocorreu entre 6 a 19 de março de 2016 em São Paulo.

Neste período de treinamento, os gestores do Hospital Regional de Quiché realizaram capacitação prática em três hospitais relacionados ao programa $\mathrm{CQH}$.

As práticas ocorreram de acordo com a formação profissional dos gestores. Assim, a gestora de pessoas do HRQ realizou treinamento no setor de gestão de pessoas. Os demais gestores realizaram treinamentos respeitando esta metodologia.

A Tabela 7 detalha a programação desenvolvida. 
Tabela 7. Cronograma da terceira capacitação realizada de 6 a 19 de março 2016.

\begin{tabular}{|c|c|c|c|c|c|}
\hline \multicolumn{2}{|c|}{ DATA } & Horario & Atividade & Palestrante & Local \\
\hline 6 de Março & Domingo & \multicolumn{4}{|c|}{ Traslado Guatemala - São Paulo Brasil } \\
\hline \multirow{8}{*}{7 de Março } & \multirow{8}{*}{$\begin{array}{l}\text { Segunda- } \\
\text { feira }\end{array}$} & $\begin{array}{l}08: 00-08: 30 \\
08: 30-09: 30\end{array}$ & $\begin{array}{l}\text { Palestra JICA-SP } \\
\text { Abertura } \\
\text { Apresentação da Metodologia } \\
\text { do III Treinamento }\end{array}$ & $\begin{array}{l}\text { Representante JICA-SP } \\
\text { Sr. Vicente Murakami } \\
\text { Dr. José Manoel } \\
\text { Dr. Haino, Dr. Milton, Sr.Antonio Carlos, } \\
\text { Enf.Isabel,Enf.Cibelle,Enf.Kyoko,Sra. } \\
\text { Mirian Nagai }\end{array}$ & HOTEL WZ \\
\hline & & $09: 30-10: 30$ & $\begin{array}{l}\text { Discussão dos Resultados do } \\
\text { Diagnostico Situacional }\end{array}$ & Antônio Carlos, Isabel, e Cibelle & \\
\hline & & $10: 30-10: 45$ & Coffee Break & & \\
\hline & & $10: 45-12: 00$ & $\begin{array}{l}\text { Apresentação Plano de Ação de } \\
\text { Melhorias das Áreas }\end{array}$ & $\begin{array}{l}\text { Apresentação:Equipe Guatemala } \\
\text { Supervisão:Antônio Carlos, Isabel, e Cibelle }\end{array}$ & \\
\hline & & $12: 00-14: 00$ & Almoço & & \\
\hline & & 14:00 - 16:30 & $\begin{array}{l}\text { Discussão dos Resultados da III } \\
\text { Avaliação modelo CQH }\end{array}$ & Antônio Carlos, Isabel, e Cibelle & \\
\hline & & $16: 30-16: 45$ & Coffee Break & & \\
\hline & & $16: 45-17: 30$ & $\begin{array}{l}\text { Orientações sobre o III } \\
\text { Treinamento } \\
\end{array}$ & Dr.Milton,Antônio Carlos, Isabel, e Cibelle & \\
\hline \multirow[b]{3}{*}{$\begin{array}{l}\text { 8/9/10/11 de } \\
\text { Março }\end{array}$} & & $08: 30-12: 00$ & Imersão Individualizada & Dr. Milton & $\begin{array}{l}\text { Complexo Hospital das } \\
\text { Clinicas da FMUSP }\end{array}$ \\
\hline & & $12: 00-14: 00$ & Almoço & & \\
\hline & & $14: 00-17: 30$ & Imersão Individualizada & $\begin{array}{l}\text { Dia } 8 \text { - Isabel } \\
\text { Dia } 9 \text { - Dr. Milton } \\
\text { Dia } 10 \text { - Dr Haino } \\
\text { Dia } 11 \text { - Cibelle }\end{array}$ & $\begin{array}{l}\text { Complexo Hospital das } \\
\text { Clinicas da FMUSP }\end{array}$ \\
\hline $12 / \mathrm{mar}$ & Sábado & & & Día de descanso & \\
\hline $13 / \mathrm{mar}$ & Domingo & & & Día de descanso & \\
\hline \multirow[t]{3}{*}{$\begin{array}{l}\text { 14/15/16/17/ } \\
\text { Março }\end{array}$} & & $08: 30-12: 00$ & Imersão Individualizada & $\begin{array}{l}\text { Dia } 15 \text { - Isabel/Cibelle } \\
\text { Dia } 16 \text { - Isabel/Cibelle } \\
\text { Dia } 17 \text { - Isabel/Cibelle }\end{array}$ & $\begin{array}{l}\text { Complexo Hospital das } \\
\text { Clinicas da FMUSP }\end{array}$ \\
\hline & & $12: 00-14: 00$ & Almoço & & \\
\hline & & $14: 00-17: 30$ & Imersão Individualizada & \begin{tabular}{|l} 
Dia 14 - Isabel \\
Dia 15 - Isabel/Cibelle \\
Dia 16 - Isabel/Cibelle \\
Dia 17 - Isabel/Cibelle \\
\end{tabular} & $\begin{array}{l}\text { Complexo Hospital das } \\
\text { Clinicas da FMUSP }\end{array}$ \\
\hline \multirow{5}{*}{$18 /$ mar } & \multirow[b]{5}{*}{ Sexta-feira } & $08: 30-12: 00$ & $\begin{array}{l}\text { Apresentações individuais sobre o } \\
\text { treinamento realizado }\end{array}$ & $\begin{array}{l}\text { Apresentação: Equipe Guatemala } \\
\text { Supervisão:Dr. José Manoel,Dr. Haino, } \\
\text { Dr. Milton, Sr.Antonio Carlos, } \\
\text { Enf.Isabel,Enf.Cibelle } \\
\end{array}$ & HOTEL WZ \\
\hline & & $12: 00-14: 00$ & Almoço & & \\
\hline & & $14: 00-15: 30$ & Avaliação do III Treinamento & \begin{tabular}{|l|} 
Dr. José Manoel \\
Dr. Haino, Dr. Milton, Sr.Antonio Carlos, \\
Enf.Isabel, Enf.Cibelle \\
\end{tabular} & HOTEL WZ \\
\hline & & $15: 30-15: 45$ & Coffee Break & & \\
\hline & & $\begin{array}{c}15: 45-17: 30 \\
\\
17: 30- \\
18: 00\end{array}$ & $\begin{array}{l}\text { Avaliação do Projeto } \\
\text { Entrega de } \\
\text { Certificados/Encerramento } \\
\text { Jantar }\end{array}$ & $\begin{array}{l}\text { Dr. José Manoel } \\
\text { Dr. Haino, Dr. Milton, Sr.Antonio Carlos, } \\
\text { Enf.Isabel,Enf.Cibelle,Enf.Kyoko,Sra. } \\
\text { Mirian Nagai } \\
\\
\text { Dr. José Manoel } \\
\text { Dr. Haino, Dr. Milton, Sr.Antonio Carlos, } \\
\text { Enf.Isabel,Enf.Cibelle,Enf.Kyoko,Sra. } \\
\text { Mirian Nagai } \\
\\
\text { Dr. José Manoel } \\
\text { Dr. Haino, Dr. Milton, Sr.Antonio Carlos, } \\
\text { Enf.Isabel,Enf.Cibelle, Enf.Kyoko,Sra. } \\
\text { Mirian Nagai e Instrutores do III } \\
\text { Treinamento }\end{array}$ & HOTEL WZ \\
\hline 19/mar & Sábado & $\begin{array}{l}\text { Traslado São } \\
\text { Paulo Brasil - } \\
\text { Guatemala }\end{array}$ & & & \\
\hline
\end{tabular}




\section{Diagnóstico situacional}

Na primeira semana de fevereiro de 2016, realizou-se diagnóstico situacional no $\mathrm{HRQ}$ conduzido pelos avaliadores Antônio Carlos da Silva e Isabel Dias.

Das oportunidades de melhoria detectadas por este trabalho, criou-se um plano de melhorias em curto prazo (quick win).

O quick win é uma ferramenta que tem como objetivo a obtenção de ganhos rápidos de forma organizada. Foi sugerida a melhora da estrutura da limpeza, higiene e organização nas áreas assistenciais (enfermarias).

\section{Disseminação do Programa CQH}

A disseminação do Programa CQH iniciou-se em 2015 através de apresentações teóricas para os hospitais das cidades de Nebaj, Joyabaj, Uspatan, Totonicapan e Solola pelos 15 gestores do Hospital Regional de Quiché. Estes hospitais são de complexidade secundária e tem como referência o Hospital Regional de Quiché para o encaminhamento de complexidades clinico-cirúrgicas.

As apresentações do Programa CQH nestes hospitais tiveram o apoio e patrocínio do Ministério da Saúde da Guatemala e foram acompanhadas por representante do órgão, Dr. Mario Gatica, vindo da capital do país.

No dia 12 de março, em reunião com a Dra. Miriam Segura, diretora do hospital e chefes dos serviços ocorreu apresentação do Programa CQH ao Hospital de Nebaj. Nesta data foi entregue também o roteiro de avaliação.

Nos dias 20, 26 de março ocorreram reuniões nos hospitais de Joyabaj e Uspatán respectivamente. Nestas oportunidades, com a participação dos diretores das unidades e gestores foram também realizadas apresentações do Programa CQH e materiais foram entregues.

No dia 16 de abril, foram realizadas reuniões nos hospitais de Totonicapan e Sololá, onde ocorreram os processos acima relatados.

Nos hospitais de Nebaj, Totonicapan e Uspatan foram realizadas avaliações nos dias 7 de maio, 15 de julho e 30 de setembro, respectivamente.

No hospital de Totonicapan, foi realizada em 28 de julho, a única reunião de consenso das avaliações realizadas nos cinco hospitais.

A Tabela 8 mostra resumo das atividades com o objetivo de disseminação do Programa CQH, conduzidas pelos 15 gestores do HRQ. 
Tabela 8. Cronograma da disseminação do Programa CQH em cinco hospitais da Guatemala.

\begin{tabular}{|c|c|c|c|}
\hline$\overline{\text { Data }}$ & Local & Participantes & Atividades \\
\hline $12 / 03 / 15$ & Hospital de Nebaj & $\begin{array}{l}\text { Dra. Miriam Segura, diretora } \\
\text { do hospital e chefes de } \\
\text { serviço (total de } 22 \\
\text { participantes) }\end{array}$ & $\begin{array}{l}\text { Apresentação teórica } \\
\text { do Modelo } \mathrm{CQH} \\
\text { Entrega do Roteiro de } \\
\text { Avaliação } \mathrm{CQH}\end{array}$ \\
\hline $20 / 03 / 15$ & $\begin{array}{l}\text { Hospital de } \\
\text { Joyabaj }\end{array}$ & $\begin{array}{l}\text { Dra. Marina Méndez, } \\
\text { diretora do hospital e chefes } \\
\text { de serviço ( } 20 \text { participantes) }\end{array}$ & idem \\
\hline $26 / 03 / 15$ & $\begin{array}{l}\text { Hospital de } \\
\text { Uspantán }\end{array}$ & $\begin{array}{l}\text { Dr. Julio López, Vice- diretor } \\
\text { do hospital e chefes de } \\
\text { serviço ( } 24 \text { participantes) }\end{array}$ & idem \\
\hline $16 / 04 / 15$ & $\begin{array}{l}\text { Hospital de } \\
\text { Totonicapán }\end{array}$ & $\begin{array}{l}\text { Dr. German Aguilar } \\
\text { Alvarado, diretor do hospital } \\
\text { e chefes de serviço ( } 37 \\
\text { participantes) }\end{array}$ & idem \\
\hline $16 / 04 / 15$ & Hospital de Sololá & $\begin{array}{l}\text { Dra. Sheily Laparra, diretora } \\
\text { do hospital e chefes de } \\
\text { serviço ( } 26 \text { participantes) }\end{array}$ & idem \\
\hline $07 / 05 / 15$ & Hospital de Nebaj & $\begin{array}{l}\text { Dra. Miriam Segura, diretora } \\
\text { do hospital e chefes de } \\
\text { serviço }\end{array}$ & $\begin{array}{l}\text { Segunda reunião e } \\
\text { realização de } \\
\text { avaliação }\end{array}$ \\
\hline $15 / 07 / 15$ & $\begin{array}{l}\text { Hospital de } \\
\text { Totonicapan }\end{array}$ & $\begin{array}{l}\text { Dr. German Aguilar } \\
\text { Alvarado, diretor do Hospital } \\
\text { e chefes de serviço }\end{array}$ & $\begin{array}{l}\text { Segunda reunião e } \\
\text { realização de } \\
\text { avaliação }\end{array}$ \\
\hline $30 / 09 / 15$ & $\begin{array}{l}\text { Hospital de } \\
\text { Uspantán }\end{array}$ & $\begin{array}{l}\text { Dr. Julio López, vice- diretor } \\
\text { do hospital e chefes de } \\
\text { serviço }\end{array}$ & $\begin{array}{l}\text { Segunda reunião e } \\
\text { realização de } \\
\text { avaliação }\end{array}$ \\
\hline $28 / 07 / 15$ & $\begin{array}{l}\text { Hospital de } \\
\text { Totonicapán }\end{array}$ & $\begin{array}{l}\text { Três representantes do } \\
\text { Hospital de Totonicapán }\end{array}$ & $\begin{array}{l}\text { Reunião de consenso } \\
\text { da primeira avaliação }\end{array}$ \\
\hline
\end{tabular}

\section{RESULTADOS}

No dia 27 de julho de 2016, ocorreu a cerimônia de encerramento do projeto "Fortalecimento de Capacidades para o Manejo de Sistemas de Gestão Hospitalar" no Hospital Regional de Quiché. O evento foi realizado no salão nobre do HRQ e contou com participação de autoridades do governo da Guatemala, Japão e do Brasil.

Como resultados do projeto ${ }^{6}$, pode-se apontar os seguintes benefícios ao Hospital Regional de Quiché:

Benefícios qualitativos: 
- Maior participação e aproximação da direção do HRQ com os trabalhadores e usuários do hospital

- Melhoria e aprofundamento de relacionamento do HRQ com o governo central

- Reconhecimento pelo Ministério de Saúde da Guatemala da evolução da qualidade médico-hospitalar do HRQ

- Acreditação como Hospital Amigo de Lactância Materna.

Benefícios quantitativos:

- Melhoria da infraestrutura com a construção de novas edificações (UTI e alojamento para acompanhantes)

- Melhoria da infraestrutura com construção de refeitório para os trabalhadores do hospital

- Melhoria da infraestrutura com reformas de edificação (enfermaria feminina)

Valorização de profissionais:

- Construção de refeitório para trabalhadores

Valorização de clientes e responsabilidade social:

- Preocupação em preservar os padrões culturais: a) introdução de práticas como o parto vertical, com o uso da roupa colorida da etnia e o acompanhamento do marido; b) prescrição do chá IXBUT - típico na comunidade e de reconhecida eficácia para melhoria do aleitamento; c) atendimento ambulatorial em idioma quiche; d) sinalização/comunicação visual em idioma Quiché e d) implantação de pesquisas de satisfação em idioma Quiché

- Realização de campanhas de conscientização junto à população escolar para doação de sangue

- Melhoria do banco de leite materno com a introdução de processo de pasteurização

- Compreensão e protagonismo do HRQ na realidade social local com a implantação de sistema de Atenção Individualizada a Vítimas de Violência Sexual

Melhoria dos processos de gestão:

- Melhoria da qualidade dos registros de reuniões

- Desenvolvimento e implantação de procedimentos operacionais padronizados (POPs)

- Adesão a protocolos da OMS

- Iniciativa em se adequar às normas de controle de radiação, mesmo não havendo legislação vigente no país

- Primeiro hospital da Guatemala a contar com incinerador de lixo hospitalar 
A evolução dos trabalhos do projeto "Fortalecimento de Capacidades para o Manejo de Sistemas de Gestão Hospitalar" não ocorreu sem sobressaltos. A substituição do diretor geral do $\mathrm{HRQ}$, Guilhermo Crocker Cordóva, durante a realização do primeiro treinamento causou apreensão quanto à continuidade do projeto. Mas o envolvimento do novo diretor, Dr. Salomon Delgado, nos trabalhos possibilitou o prosseguimento do projeto e os resultados obtidos.

As três substituições de ministros da saúde da Guatemala durante o transcorrer do projeto também causaram dúvidas quanto à manutenção dos trabalhos. $O$ projeto iniciou-se na gestão do Dr. Jorge Alejandro Villavicencio, que foi seguido pelos Drs. Luis Enrique Monterroso e Mariano Rayo. O final do projeto ocorreu durante a gestão da Dra. Lucrecia Mack. Em relação ao Ministério da Saúde da Guatemala, pôde-se observar apoio para o desenvolvimento do projeto. Muitas atividades do projeto contaram com a participação de representante do Ministério da Saúde.

Os resultados da disseminação do Programa CQH para cinco outros hospitais da Guatemala foram aquém do esperado. $O$ desencontro nos trabalhos, observado entre a primeira e a segunda avaliação, somado à diminuição do número de gestores envolvidos no projeto afetou o cronograma planejado para os trabalhos, com prejuízo maior no desenvolvimento dos trabalhos para a disseminação do modelo $\mathrm{CQH}$ aos cinco hospitais.

Os gastos com as ações do projeto exigiram o dispêndio de $¥ 25.332 .000$, cerca de US $\$ 250.000$, pelo Governo Japonês. Este montante foi utilizado para despesas de viagem, hospedagem e alimentação dos participantes. Neste montante estão inclusos ainda despesas de tradução de livros e manuais de treinamento doados pelo Programa CQH e PROAHSA.

A contraparte guatemalteca foi na ordem de 386.982 quetzales, cerca de US\$ 48.000, para aquisição de equipamentos de informática e despesas de viagem para a disseminação do Programa CQH para os hospitais de outras cidades.

Os voluntários do PROAHSA e o CQH doaram cerca de 720 horas entre planejamento, treinamento, monitoramento de visitas hospitalares e realização de avaliações ao $\mathrm{HRQ}$.

A metodologia de relacionamento entre as entidades participantes foi realizada através de entendimento direto com a JICA, por meio dos escritórios do Brasil e da Guatemala, sob a supervisão do PROAHSA e CQH.

O governo japonês aprovou o prosseguimento dos trabalhos para a melhoria da gestão hospitalar do Hospital Regional de Quiché até o ano de 2020. Para a continuidade das ações, existe orçamento de US\$1.000.000.

O prosseguimento dos trabalhos passará a ocorrer por meio de projeto tripartite governamental. Assim, ocorrerá o envolvimento da Agência Brasileira de Cooperação do Ministério das Relações Exteriores do Brasil, como órgão coordenador pelo lado brasileiro. 


\section{CONCLUSÃO}

Após dois anos de desenvolvimento do projeto, o Hospital Regional de Quiché tem ainda caminho a percorrer para atendimento aos requisitos de excelência e desempenho.

Porém, é inegável a evolução positiva observada na gestão do hospital.

Os resultados deste projeto permitem visibilizar a validade da implantação de um modelo de gestão para a melhoria da qualidade hospitalar. O projeto permitiu ao HRQ conhecer, avaliar seus processos de gestão e, por meio destas avaliações, buscar a melhoria contínua de seus serviços.

Apesar do resultado relacionado à disseminação do Programa CQH para outros cinco hospitais da Guatemala ter sido comprometido, espera-se que os resultados positivos observados no $\mathrm{HRQ}$ sensibilizem outros hospitais a implantar o Programa CQH, a fim de obter melhoria da qualidade.

A parceria entre o PROAHSA, cedendo especialistas em administração hospitalar para as capacitações, e o CQH, compartilhando o modelo de gestão, possibilitaram os resultados obtidos.

O patrocínio pela agência de cooperação do governo japonês (JICA) arcando com os custos de viagem, hospedagem e alimentação viabilizou a consecução dos trabalhos desenvolvidos no Brasil e na Guatemala.

Conclui-se pela importância de projetos desta natureza ao observar-se a evolução positiva do HRQ. Ao cabo de dois anos de desenvolvimento do projeto, o $\mathrm{HRQ}$ assumiu protagonismo dentre os hospitais públicos da Guatemala.

\section{REFERÊNCIAS}

1. CQH: Faça Parte [página na Internet]. São Paulo: Programa CQH; c20062017 [visto em 2017 março 03]. Disponível em:

http://cqh.org.br/portal/pag/area.php?p_narea $=97$

2. Hospital das Clínicas: Home PROAHSA. São Paulo: HCFMUSP; c1997 [visto em 2017 março 03]. Disponível em

http://hcnet.usp.br/index.php?option=com_content\&view=article\&id=418:pro ahsa-menu\&catid=20: conteudo

3. JICA Representação no Brasil: Sobre. São Paulo: JICA; c2017 [visto em 2017 março 03]. Disponível em:

https://www.jica.go.jp/brazil/portuguese/office/about/index.html

4. Becerril-Montekio Víctor, López-Dávila Luis. Sistema de salud de Guatemala. Salud pública Méx [revista na Internet]. 2011 Ene [citado 2017 Abr 11] ; 53( Suppl 2 ): s197-s197. Disponible en: 
http://www.scielo.org.mx/scielo.php?script=sci_arttext\&pid=S003636342011000800015\&lng=es

5. FNQ: Melhores em Gestão. São Paulo: FNQ; c2015 [visto em 2017 abril 10]. Disponível em http://www.fnq.org.br/avalie-se/melhores_em_gestao

6. JICA. Informe de Finalización del Proyecto Fortalecimiento de Capacidades en el Manejo de Sistemas en Gestión Hospitalaria - Periodo: Julio 2014 Julio 2016; 2016

Recebido: 10/04/2017; Publicado: 17/04/2017

Correspondência: Milton Osaki. E-mail: m.osaki@yahoo.com.br

Conflito de Interesses: o autor não declarou conflito de intereses

(C) This is an Open Access article distributed under the terms of the Creative Commons Attribution License, which permits unrestricted use, distribution, and reproduction in any medium, provided the original work is properly cited. 